\title{
The Aurora Polaris and the Light of the Night Sky
}

$\mathrm{T}$ $\mathrm{HE}$ relation of the aurora to the light of the night sky formed the topic of a Geophysical Discussion at the Royal Astronomical Society on May 19, under the chairmanship of Lord Rayleigh. Prof. S. Chapman opened the discussion.

Owing to its often striking appearance, the aurora polaris has long been studied. Dr. J. M. Stagg gave an account of its appearance and changes as observed at Fort Rae, near the auroral zone, during the second International Polar Year (1932/3); one remarkable feature he described was the occasional repetition, on two successive nights, of unusual and distinctive auroral appearances.

The situation of auroral arcs and rays and sheets and diffuse clouds has for many years been determined with considerable accuracy by the methods introduced by Prof. C. Störmer, whose long-continued and fruitful work was rewarded, not long since, by the discovery that the very high auroras, extending up to $600 \mathrm{~km}$. or even more, are generally in the part of the atmosphere which, though visible from the ground after sunset or before sunrise, is still illumined by the sun's rays.

Naturally, the auroral light was also examined by the spectroscope ; the spectrum includes several bands, some of which have long been known to be the so-called negative bands emitted by excited positive molecular ions of nitrogen $\left(\mathrm{N}_{2}{ }^{+}\right)$; in addition, it contains a bright yellow-green line.

A similar line was found to be present in the light of the night sky. But in other respects this light differs considerably from the auroral light, as was first clearly emphasized by Lord Rayleigh ; it does not contain the $\mathrm{N}_{2}+$ bands : it is diffuse and formless, but always present, in all latitudes ; its variations are much less rapid and less great than those of the aurora.

The wave-length of the green line in the nightsky spectrum was determined very accurately in 1923 by Babcock, using an interferometer; it is $5577 \cdot 35$, and its width is very small, only $0.030 \mathrm{~A}$. A few years ago, Harang and Vegard (who has been one of the most assiduous and successful workers on the auroral spectrum) determined the wave-length of the green auroral line in the same way, and thus proved that the line is the same in the two spectra. These interferometer measures show that the line is of atomic origin and not part of a molecular band. Harang and Vegard have also demonstrated the atomic character, and found the accurate wave-lengths, of two red lines, 6300 and 6364 , in the auroral spectrum.
The same two lines, and also the yellow doublet of neutral atomic sodium, have been observed interferometrically in the night-sky spectrum; this was done in France, where several spectroscopists of the highest skill-Cabannes, Dufay, Gauzit and Bernard--are studying this light. The lines named above are the only ones in the auroral or night-sky spectra the atomic character of which has been established by use of the interferometer. The suspected presence of a line due to atomic nitrogen has not yet been confirmed or disproved by this method.

Recent years have seen a great advance in the accuracy with which the lines and bands in the two spectra are measured, and in the range of the spectrum observed. For the night sky, the observations have been extended far into the ultra-violet (notably by Gauzit) and into the infra-red (by Slipher). Gauzit has made the remarkable discovery that the night-sky spectrum extends to at least $2950 \mathrm{~A}$. in the ultra-violet; he suggests that this light comes from the lower part of the ozone layer, which would absorb such light if it came from above (as it absorbs the sunlight of that wave-length, during the day).

The origin of the green line 5577 long remained mysterious, and it was not produced in the laboratory until 1925, when McLennan, with Ireton, and others of his Toronto fellow-workers, began a brilliant series of researches which, aided by other discoveries of the time, by Frerichs, Rubinowicz, and Bowen, established that the line is due to neutral atomic oxygen. The atomic transition involved is from the metastable ${ }^{1} S_{0}$ state, with a mean life-time of about 1 second, to a ${ }^{1} D_{2}$ state, lifetime about 100 seconds, these times having been calculated by Stevenson; transitions from the latter state to the (multiple) ground state ${ }^{3} P_{012}$ give the red lines 6300,6364 already mentioned. Thus the nature of the atomic lines in the auroral and night-sky spectra is now definitely ascertained.

Kaplan's laboratory experiments on nitrogen mixed with oxygen have closely reproduced the two spectra, and in particular have led to the recognition and understanding of some of the principal (Vegard-Kaplan) bands in them. Though some important bands still remain unidentified, it seems likely that all the principal bands, in both spectra, are due to molecular nitrogen (neutral in the night-sky spectrum, and both neutral and ionized in the auroral spectrum). The two spectra differ greatly in the degree of excitation involved, that of the night-sky spectrum (about 7 volts) 
being much less than for the auroral spectrum (about 20 volts).

Dr. W. C. Price described the energy-level diagrams for nitrogen and oxygen, indicating the nature of the various nitrogen emission bands, and also explained how the oxygen molecules, but not the nitrogen molecules, become dissociated by sunlight in the high atmosphere; both also become partly ionized, the nitrogen in the $F$ layer and the oxygen $\left(\mathrm{O}_{2}\right)$ in the $E$ layer. The energy of the night-sky light probably comes from the re-combination of some of the oxygen atoms throughout the night, and Chapman has outlined the processes by which this energy can be transformed into the observed light. The auroral light is probably due to the impact upon the molecules in the high atmosphere of fast-moving particles coming from the sun.

The temporal variations in the intensity of the night-sky light have been studied in a remarkable series of researches, carried on for eleven years under the exceptional difficulties of English skies, by Lord Rayleigh. He devised a simple instrument, which was described by the Astronomer Royal, containing a permanent weak comparison light-source (a radioactive uranium salt) and which by means of six simple colour filters enabled the intensity of the faint night-sky light, in three spectral regions, to be estimated within a range of no fewer than thirteen grades. Copies of this instru- ment were used by other observers, and notably for about eight years at the Cape and Canberra Observatories, in a co-operative research organized by Lord Rayleigh. Variations of annual, semiannual and eleven-year (sunspot) periods were observed, as well as irregular day-to-day changes. McLennan, and independently Lord Rayleigh, also found a variation during the night, with maximum at 12 or 1 p.m., which has since been confirmed also by Russian observers. In addition, Lord Rayleigh made the very difficult determination - of great theoretical interest-of the absolute photometric intensity of the green nightsky light (5577); this indicated that $1.8 \times 10^{12}$ atomic oxygen transitions $\left({ }^{1} S_{0}\right.$ to $\left.{ }^{1} D_{2}\right)$ occurred per second in each square centimetre column of the atmosphere.

In Lord Rayleigh's own contribution to the discussion, he stressed the difficulty of work on the night-sky spectrum, owing to its faintness, which makes it impossible, in the ordinary way, to get luminous spectra with much dispersion, unless large and expensive prisms are used. $\mathrm{He}$ considered that his father's tube-spectroscope might find a useful application in this field; this instrument consists of a tube, with lenses at each end, which contains numerous, perhaps twenty, glass prisms in a liquid, carbon disulphide, of very similar refractive index; it can give great dispersion with little loss of light.

\section{Obituary Notices}

Sir Robert Bolam, O.B.E.

$\mathrm{T}$ HE death of Sir Robert Bolam, which took place at his home in Newcastle-upon-Tyne on April 28, not only removes an outstanding personality of the medical profession from the north of England, but also one who by reason of his activities in larger spheres of medical interest had made a name known and respected in the profession all over Great Britain.

Bolam's name will always be connected with the two bodies to which he gave such long and valued service, the General Medical Council and the British Medical Association. He was first a member of the General Medical Council in 1920, being elected as a direct representative for England; then from 1928 until his death he represented the University of Durham. It was only natural that his acumen and capacity for attention to detail led ultimately to his appointment as Chairman of Business, and this no doubt, had he lived, would have been followed by yet higher honour in the Council.

In the British Medical Association, Bolam was an active member for forty years, and here again his administrative ability, shrewdness and clear thinking soon brought him into prominence. He became a member of Council in 1915, acted as chairman of the Journal Committee and of the Hospitals Committee ; finally in 1920 he became chairman of the Council. His energy and initiative were responsible for the transfer of the Association's headquarters in London from the Strand to its present site in Tavistock Square. In this move to what is now rapidly becoming the intellectual quarter of London, Bolam displayed foresight and faith which have since been justified abundantly.

In spite of the energy which Bolam expended in the services to the General Medical Council and the British Medical Association, either of which would have been an ample occupation for a man of normal activity and talents, he gave his best services over a long period of years and in many capacities to his old school, the University of Durham College of Medicine and the associated Royal Victoria Infirmary. The posts he held were various; in the College of Medicine he was in turn lecturer on physiology and pathology, joint lecturer on physiological physics and chemistry, professor of medical jurisprudence, subcurator of the museum, while in the Infirmary he was assistant physician and pathologist. Later he 